\title{
Analysis on the Design and Application of the Small and Large Current Photomultiplier Tube
}

\author{
Yanmin Zhou ${ }^{1, a}$ \\ ${ }^{1}$ Chongqing College of Electronic Engineering, Chongqing 401331, China \\ azhouyanmin023@126.com
}

Keywords: Small, Large current, Photomultiplier tube, Design

\begin{abstract}
The photocell and photomultiplier has become a piece of very important photoelectric conversion device and photoelectric emission device, it plays a decisive role in optoelectronic devices. The photomultiplier tubes can be applied in the field of the weak light signal detection, and is the core of a variety of precision measuring instrument and device in high energy physics, satellite remote sensing survey, geological prospecting, biological, medical, military reconnaissance, spectral analysis and other fields that has important significance. Correctly grasping the characteristics of photocell and photomultiplier tubes, plays a key role for the correct use of photoelectric equipment. We will be focused on the principle and characteristics of the small and large current photocell and photomultiplier.
\end{abstract}

\section{Introduction}

The photomultiplier tube is a kind of based on the photoelectric effect, secondary electron emission and electron optical theory, on the basis of the incident light into a weak photoelectron and multiplication of photoelectric detector, has been in the field of weak optical signal detection and its important status, is the core of a variety of precision measuring instruments, the device is widely used in high energy physics, spectral analysis, remote sensing satellite measurements, chemical, geological exploration, medical, biology, medicine, military reconnaissance and environmental monitoring, etc. In the physics experiment teaching, a lot of experiment content involves the use of the photomultiplier tube, but the students know about its working principle and method of use, especially the spectral characteristics of photomultiplier tube, is a difficulty of experiments. Based on this, through to the monochromatic, thermal probe preferential selection, experiment research plan meticulously, optimization design of the photomultiplier spectrum characteristic experiment, students can not only complete the photomultiplier relative spectral response curve measurement, can also study seam width, temperature, magnetic field, such as working voltage on the photomultiplier tube the influence of the spectral characteristics, can also use this experimental platform, other spectral characteristics of photoelectric devices, deepen students of photomultiplier working principle, the understanding of spectral characteristics. The experiment has as a comprehensive, design and research experiment content in university physics experiment.

\section{The Working Principle and Characteristics}

The photoelectric effect is an important phenomenon in physics. Under the irradiation of light, the electrons within the certain substances to be photon stimulated current formation, which formed the light electricity. Light on the surface, it can be seen as an object by a series of energy for the $\mathrm{E}$ to the size of the photons illuminate, the electrons within the material being stimulated photons, and a current, namely the light electricity. In beam, the energy is proportional to the frequency of light photons have, if, in the metal free electron absorbs a photon energy, absorption of energy is equal to or greater than the metal work function, the electron will be emitted form a photoelectron. When the beam of the photon energy is not enough to make the electronic escape away, won't produce current.

Light on surfaces is after the photoelectric effect. Usually, we put the photoelectric effect into three broad categories: photoelectric effect, the photoelectric effect and semiconductor optical volts 
effect. Under the effect of light, the electrons are inspired and transgression surfaces, this phenomenon is called the photoelectric effect, there are also call it the photoelectric emission effect, based on the photoelectric effect outside there are a lot of photoelectric component, typical device with photocell and photomultiplier tubes.

The Photocell Principle and Characteristics. Cell is a kind of optical signal to electrical signal conversion of sensor devices, photovoltaic cells based on the photoelectric effect, and has many good characteristics: good spectral characteristics, high sensitivity, stable performance, long service life, etc. Photocell application is very extensive, used in solar cell research, many aspects, such as optical fiber communication and automation control. Cell consists of a cathode and an anode, and sealed in a vacuum glass tube. Cell cathode material largely determines the performance characteristics of photocell. In the study of the basic characteristic of the photocell, mainly studies its volt-ampere characteristics, light characteristics and spectral characteristics, etc.

In the experiment of measuring the characteristics of the photocell, with mercury materials as light source, in order to get a different frequency of the incident light filter is adopted, the distance between light source and the photocell is $250 \mathrm{~mm}$. In order to get the accurate parameters of the results, the experiment equipment must be between coaxial contour, equipment set up later, began to set the Angle between the voltage and the Polaroid alpha at the ends of the cell, based on photoelectric cell characteristics of experimental measurement.

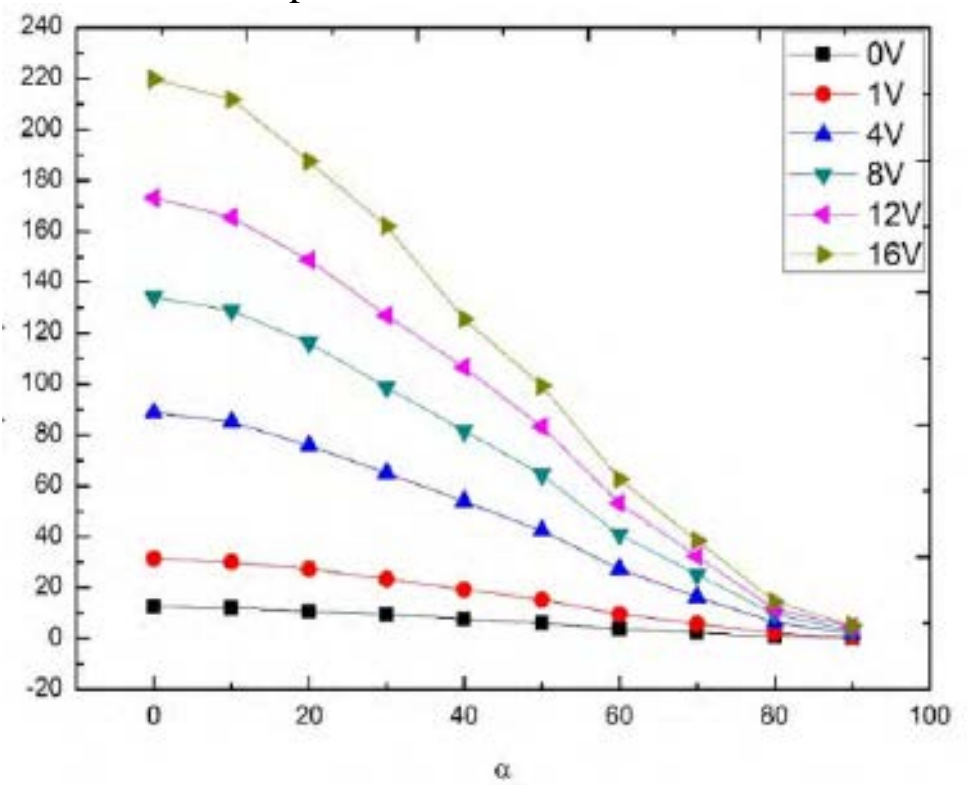

Fig. 1 photocell light characteristic curve

The Principle and Characteristics of Photomultiplier Tube. Photomultiplier tube is a kind of has the very high internal gain photosensitive device, which USES between the semiconductor and metal outside of the photoelectric effect. Photocell is composed of three parts, the first part is made up of the photocathode with photoelectric emission materials, the second part is made up of a series of double electrode, and the third part is the ultimate collection of electronic anode. First of all, the photocathode receives the light after injection of photoelectron, coated with the multiplication of secondary electron emission materials has been photoelectron bombardment can produce multiple secondary electron, photomultiplier tubes within multiple multiplication, between each this imposed certain voltage, the voltage of the role is to accelerate the production of the secondary electron and so on, which are an increasing number of photoelectron, photoelectron agglomeration and the pulse signal, finally the pulse signal to the anode. When the light is very weak, the photocurrent generated by photoelectric cell is very small; using a photomultiplier tube can improve the sensitivity. Photomultiplier tube is a kind of incident light into a faint can convert the photoelectron, and still can obtain high multiplication factor is an important photoelectric detector. It relies mainly on the secondary electron emission, the photoelectric effect and electron optical theory, is an important photoelectric emission devices. 


\section{The Structure of the Small and Large Current Photomultiplier Tube}

Photomultiplier tube by photoelectric emission cathode (photocathode) and electron optical input system (between photocathode and the first double pole D1 system), the secondary emission multiplication system and electronic collection pole (anode) and so on, is a vacuum device. The collection of the electronic optics system rate above $85 \%$ and discreteness of transit time (the photoelectron emitted by each point refers to the cathode surface to achieve the first double pole everywhere on the time difference) about $10 \mathrm{~ns}$. Best performance electron optical systems used the photocathode spherical shape, and additional 3 cylindrical electrode. In this way, the cathode surface potential distribution more uniform, and from the center and edge of cathode emission electron trajectory length difference is rather small, can make the discreteness of transit time is close to zero. The multiplication of photomultiplier tube pole structure is divided into focus and not focus on two. Non focus with louver structure and box - net room structure of two; Focus type have straight type structure and two round cage focus structure.

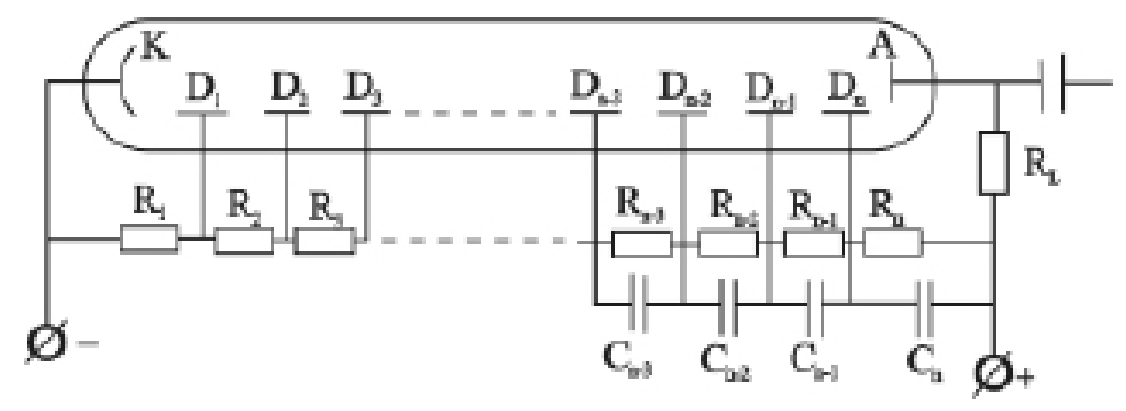

Fig. 2 photomultiplier tube voltage divider design principle

Within a certain range, the photomultiplier tube plate output current and incoming there is better linear relation between light intensity. Photomultiplier tube linear range is large, the anode output dc current linear range can cross 4-5 orders of magnitude, larger range of pulse conditions. When the light intensity is increase, the output current will gradually deviate from the straight line. Usually we put the deviation from linear corresponding linear current value will be 5\% or $10 \%$ points is defined as the maximum linear current. It can be roughly thought that in the biggest linear electrical work flow, anode than normal and low sensitivity of $5 \%$ or $10 \%$.

\section{The Nonlinear Digital Correction of Photomultiplier Tube}

According to the previous discussion, within a certain range, the nonlinear photomultiplier tubes, but the anode output current as a function of input light intensity is fixed, can pass correction, extended linear current range.

At present, the use of digital oscilloscope brought great convenience to the data analysis and processing. Multiplier output and input measurement by tracing point method, the relationship between general tracing point method is to use two photomultiplier tubes, one is the measured tube, another control light intensity, make its work in the linear area, known as reference. Pulse generator drives highlight, highlighting diodes respectively on the pipe and the reference under test. In order to ensure the accuracy of measurement, using photocell is as reference. Photocell generally can reach more than 1 A linear current; can guarantee the linear current in measurement range. To test the output of the pipe and the reference signal recorded by digital oscilloscope. Every time a square wave pulse, record the two output signals. Through the analysis of the data, we believe that the nonlinear effect of multiplier effect is small, negligible; oscilloscope response is the major influencing factors. Oscilloscope every gear is a response to waveform signal output to, in its response to the following, although there is a signal, but no output. Refer to the minimum response of tube and tube under test position is different, causing the waveform is zero, however, it makes the linear intercept is negative. In addition, the scope baseline some ups and downs will affect reading, cause data of ups and downs, this fitting will also affect to the data. 


\section{The Application Development and Outlook of Photomultiplier Tube}

The photomultiplier tube as the weak light detector development has been decades of history, its application is becoming more and more widely, in optical physics, automation control, space exploration and biological science and technology are obtained the promotion and application. With the development of science and technology, the development of the photomultiplier faster and faster, with its basic process continuously improve, gradually increase performance parameters, many special functions and special structure of photomultiplier tubes are constantly emerging.

Any creature, different species, different light intensity, are generally very weak, light intensity between a few to several hundred photons, belongs to the weak light. Some biological under normal conditions of weak light intensity is constant, in the event of lesions, the faint light of will change significantly. Medical use this property to make bioluminescent measuring instrument to detect whether the biological lesions occur, because bioluminescent is very weak, bioluminescent probe made of dark room form of measuring instrument, photomultiplier tubes are the main components.

More anode photomultiplier tube as a photoelectric sensing device, because of the compact space structure, the characteristics of high gain, low noise, in the array detector and position sensitive detector is widely used, especially the metal channel multiplication pole structure, multiple anode photomultiplier performance is improved greatly, the crosstalk between each road can be less than $2 \%$, is increasingly used as a readout device of array detector.

This response rate is $18 \mathrm{~mm}$ in diameter of the photocathode, its dark count rate is less than 200 times per second, it combines high gain, single electron resolution, response time, and linearity of the pulse at a suit, red light has a high sensitivity, high response rate, large cathode area and outstanding ability of photon counting these features make it very suitable, near infrared spectrum detection and chemical luminescence and bioluminescent weak light detection applications.

\section{Summary}

Through measurement of photocell photoelectric properties and volt-ampere characteristics of photomultiplier tube light, spectral response and volt-ampere characteristics of experimental measurement, insight into the photocell and photomultiplier related characteristic curve changes, has important significance for the correct use of photoelectric devices. Looking to the future, photomultiplier tubes will be in the design of special structure, improve the technical performance of the conventional tube type, continuous innovation to adapt to the special features, etc.

\section{References}

[1] O','’Neill,P. M.Badhwar-O',',Neill galactic cosmic ray model update based on advances composition (ACE) energy spectra from 1997 to present. Advances in Space Research . 2011(08).

[2] J. B. Parkinson,L. W. Morton. Radiation Testing of the W71 Star Sensor Photomultiplier Tube. Aerojet-General Corporation Report 160:3741X6439 . 2012(10).

[3] Donald F Heath, Paul A. Sacher. Effects of a Simulated High-Energy Space Environment on the Ultraviolet Transmittance of Optical Materials between 10501K and 3000k. Applied Optics . 2012(12).

[4] Gilbert A. Haynes, Wm. E. Miller. Effects of 1.2 and $0.30 \mathrm{MeV}$ Electrons on the Optical Transmission Properties of Several Transparent Materials. NASA Technical Note TN D-2620 . 2011(08).

[5] Lemaire J, Johnston A D, Heynderickx D, et al.TREND22 (Trapped Radiation Environment Model Development) Final Report. European Space Agency Contract Report , ESTECП Contract No. 9828П92ПNLПFM . 2012(10). 